\title{
Bain circuit occluded by foreign body
}

The purpose of this report is to describe a potentially hypoxic event which occurred during mask induction with the Bain circuit in a healthy, ASA I boy. Failure of induction occurred because a pop-off weight from a bubble bottle was lodged in the elbow connecting the Bain circuit to the mask and prevented gas flow. Pethick and Foëx-Crampton Smith tests of the Bain circuit could also have missed this condition. Such an anaesthetic mishap could occur with any breathing circuit which is re-used. Although there was no serious misadventure, the potential for an hypoxic complication with either intra-venous induction of anaesthesia, or dislodging of the weight into the airway was significant. We conclude that the standard tests of system patency do not ensure safety.

Cette observation décrit une cause potentielle dhypoxie survenue pendant linduction avec un circuit Bain, au masque, de l'anesthésie chez un jeune garçon ASA I. La présence dans le raccord coudé de branchement entre le circuit Bain et la masque d'une pesée de surpression provenant d'une bouteille de barbotage obstrue le débit des gaz. Les tests de Pethic et Foëx-Crampton sur le circuit Bain n'auraient pas décelè cette anomalie. Ce type de problème peut survenir lorsqu'un circuit anesthésique est réutilisé. Bien quîci on ne puisse déplorer de complication grave, la possibilité d'une hypoxie lors d'une induction intraveineuse ou par migration du corps étranger dans les voies aériennes est réelle. Nous concluons que les test standards d'intégrité du circuit n'assurent pas une complète sécurité.

The Bain circuit ${ }^{1}$ is a modification of the Mapleson D system in which fresh gas flow is delivered at the end nearest the patient through a small inner tube located within a larger corrugated tube. It has several advantages over the circle system in that it is light-weight, convenient and has no valves. The major disadvantage is the re-

\section{Key words}

EQUIPMENT: circuits, Bain; HYPOXIA.

From the Department of Anaesthesia, University of Western Ontario, Victoria Hospital, London, Ontario.

Address correspondence to: Dr. P. Lindsay, Department of Anaesthesia, University of Western Ontario, Victoria Hospital Corporation, London, Ontario N6A 4G5.

Accepted for publication 21st October, 1993. quirement for high gas flow rates to prevent re-breathing. It is used extensively at the University of Western Ontario for both adult and paediatric anaesthesia. Although most problems with the circuit involve disconnections, obstruction problems related to kinks in the gas delivery tube have been reported. ${ }^{2}$ We would like to report an anaesthetic incident involving obstruction of the Bain circuit with a foreign body.

\section{Case report}

A two-year-old boy, ASA I, presented as a fasting outpatient for elective repair of a right inguinal hernia under general anaesthesia. Past medical history was noncontributory with respect to cardio-pulmonary disease. The child had no known allergies and was not receiving any medications. There was no family history of anaesthetic complications. On examination he was of appropriate weight and appearance for his age. The chest was clear to auscultation. Heart sounds were difficult to assess because of his agitated state.

A disinfected Bain circuit had been attached to the anaesthetic machine and visually inspected to insure that all connections were properly applied. No other specific tests of the circuit were performed. Drugs were drawn up before the patient was brought into the $O R$. The plan was to carry out an inhalational induction initially with $\mathrm{N}_{2} \mathrm{O}, \mathrm{O}_{2}$ and then adding halothane. A pulse oximeter was the only initial monitor. It was planned that ECG, temperature and non-invasive blood pressure monitoring and the addition of $\dot{v}$ access would be initiated after induction of anaesthesia. An ilio-inguinal/ilio-hypogastric nerve block with bupivacaine was to be performed for postoperative analgesia and to minimize vapour requirements.

After approximately $60 \mathrm{sec}$ of attempted mask induction with $\mathrm{N}_{2} \mathrm{O}, \mathrm{O}_{2}$ and halothane the child showed no signs of being anaesthetized. He remained well oxygenated, did not demonstrate stridor and showed equal chest wall expansion. The reservoir bag was somewhat inflated but was not moving with ventilation and there was no detectable odour of halothane in the room. Although the patient was still struggling it was felt that there was an adequate seal with the face mask. The gas flow settings were rechecked as were all connectors from the anaesthetic machine to the Bain circuit as well as the circuit itself. 
The source of the obstruction did not become apparent until the inside of the face mask was examined. The plastic elbow connecting the Bain circuit to the face mask was occluded by a cylindrical metal object (see Figures $I$ and 2). When this was removed by vigorous shaking, halothane was able to reach the patient and he was soon anaesthetized. The remainder of the anaesthetic continued as planned without further mishap.

\section{Discussion}

Most reported problems with the Bain circuit involve disconnections of the inner tube rather than complications related to obstruction. ${ }^{2}$ Eisenkraft and Sommer ${ }^{3}$ reviewed three tests which should be routinely performed prior to using each Bain circuit: the Foex-Crampton Smith test, ${ }^{4}$ the Pethic test ${ }^{5}$ and assessment of the pressure release valve. ${ }^{6}$ Although they were not carried out in the present case, it is possible that the occluding cylinder would not have been discovered in any case. The Foex-Crampton Smith test consists of setting the oxygen and air flow at an arbitrary flow rate so that the rotameters are elevated and then occluding only the small inner tube and watching for descent of the rotameters. This test ensures that there is no leak in the delivery system from the flowmeters to the end of the breathing circuit. If such a leak were present then the increase in back pressure would escape and the rotameters would not descend.

The Pethic test is a more specific test of the integrity of the small inner gas delivery tube of the Bain circuit. Increasing the gas flow via the oxygen flush valve without occlusion of the delivery tube causes the reservoir bag to deflate by a Venturi effect created at the open end of the Bain circuit. A leak within the delivery tube would allow gas to escape into the outer tube and thus prevent the reservoir bag from deflating.

Finally, assessment of the pressure release valve is performed as suggested by Seed. ${ }^{6}$ The outer tube of the Bain circuit is occluded while flushing the inner tube with oxygen. This causes gas to flow out of the inner delivery tube and back into the reservoir via the outer corrugated tube. As pressure increases in the reservoir bag, the pressure release valve should open at approximately $50 \mathrm{~cm}$ of $\mathrm{H}_{2} \mathrm{O}$ and the pressure gauge should not increase further despite continued flushing.

All three of these tests. can be performed without the elbow connecting the Bain circuit to the mask or endotracheal tube. Certainly the Foex-Crampton Smith test must be performed without the elbow in place. When the Pethic test was performed on our circuit with the occluded elbow in place, the bag did expand. Simple visual inspection of the Bain circuit and elbow should have picked up the occluding cylinder. However, when we asked other anaesthetists to examine the tube without

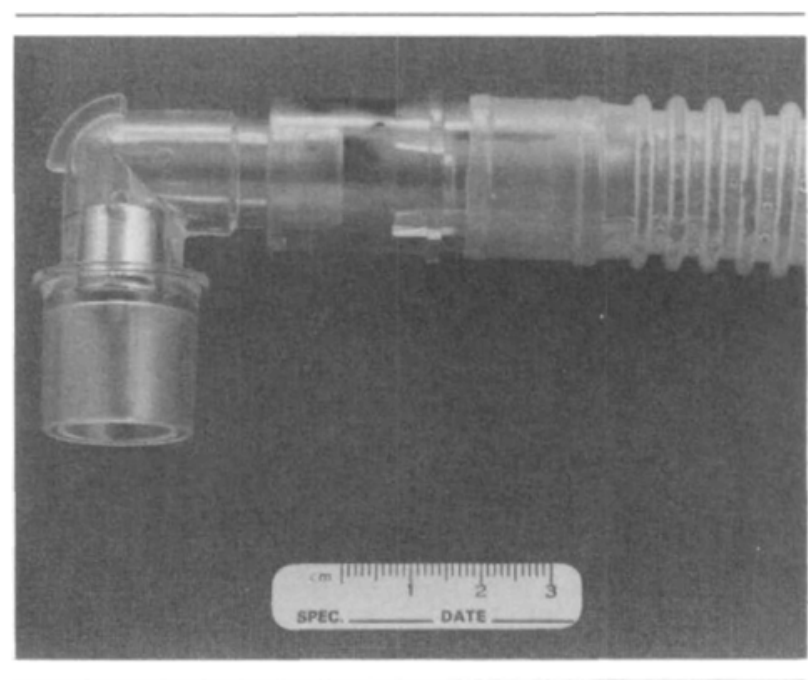

FIGURE 1 The end of the Bain circuit with the pop-off weight lodged in the elbow.

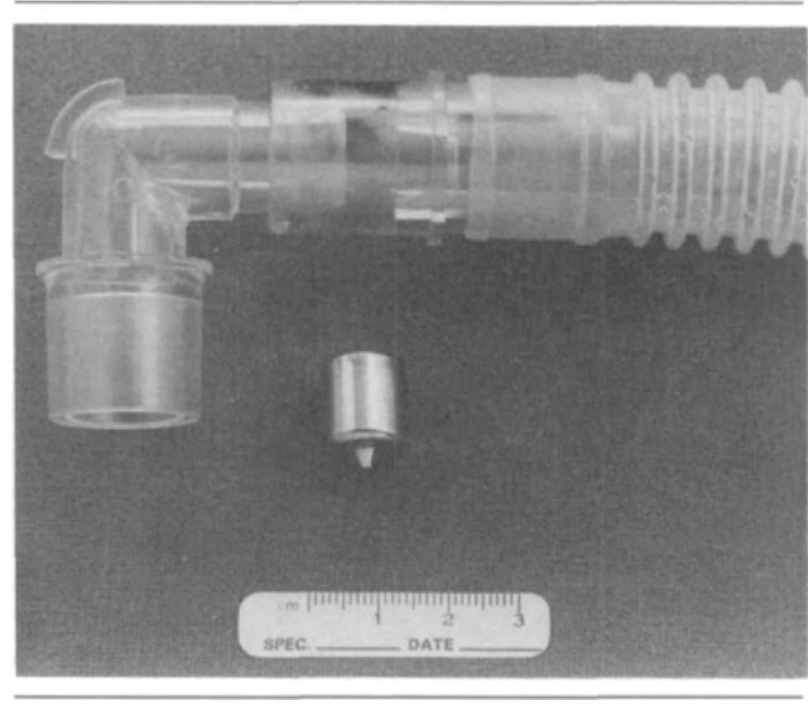

FIGURE 2 Pop-off weight removed from the elbow of the Bain circuit.

being told of the problem, it was missed on all but the most thorough examination. A kinked inner tube causing obstruction in a Bain circuit was reported by Mansell.? Again, this resulted in airflow obstruction and was only visible when the circuit was examined under strong light despite the transparent outer tube.

The source of the metal cylinder was later discovered to be a pop-off weight from a bubble bottle used to humidify the wall oxygen. How it got into the tube is not known. The Bain circuits are disinfected in hospital after each use and are used for approximately one month. The disinfection process involves disconnecting all circuit components and placing them in a wash tub. The Bain circuits are not washed in isolation of other valve and 
gas connection systems. The loose components are then placed in bags and reassembled by the respiratory technicians before use. The pop-off weight is normally retained within the lid of the bubble bottles and is not washed separately. It is likely that the restraining cap over the weight came off during the washing process and the weight then became lodged in the elbow of the Bain circuit.

Although there was no serious misadventure in the present case, the potential for an hypoxic complication is considerable. There was a risk of the cylinder becoming dislodged and falling into the patients' mouth and causing airway obstruction. If intravenous induction of anaesthesia had been attempted then considerable delay in connecting the Bain circuit to the endotracheal tube could also have taken place. This mishap could have occurred with any anaesthetic circuit which is re-used. We would like to emphasize that both the Pethic ${ }^{5}$ test and the occlusion test suggested by Seed ${ }^{6}$ be performed with the elbow in place to test the entire circuit. As we were able to demonstrate, the Pethic test missed the obstruction when performed without the elbow, but did demonstrate obstruction with the elbow in place. Furthermore, we would suggest that opaque components not be used in either a Bain circuit or a circle system.

\section{Acknowledgements}

The authors wish to express their gratitude to Dr. J. Bain for his helpful suggestions and to Sue Davey for her expert secretarial assistance in the preparation of this manuscript.

\section{References}

1 Bain JA, Spoerel WE. A streamlined anaesthetic system. Can Anaesth Soc J 1972; 19: 426-35.

2 Wyant GM. Mechanical Misadventures in Anaesthesia. Toronto: University of Toronto Press, 1978.

3 Eisenkraft JB, Sommer RM. Equipment failure: anaesthesia delivery systems. In: Benumof JL, Saidman LJ (Eds.). Anesthesia and Perioperative Complications. Toronto: Mosby Year Book, 1992: 77-127.

4 Foëx P, Crampton Smith A. A test for co-axial circuits (Letter). Anaesthesia 1977; 32: 294.

5 Pethick SL. (Letter) Can Anaesth Soc J 1975; 22: 115.

6 Seed RF. A test for co-axial circuits. More questions and further suggestions (Letter). Anaesthesia 1977; 32: 676-7.

7 Mansel WH. Bain circuit: "The hazard of the hidden tube." Can Anaesth Soc J 1976; 23: 227. 